\title{
Global Behavior of a Nonlinear Difference Equation with Applications
}

\author{
Decun Zhang, Jie Huang, Liying Wang, Wenqiang Ji \\ Institute of Systems Science and Mathematics, Naval Aeronautical and Astronautical University, Yantai, China \\ Email: dczhang1967@tom.com,ytliyingwang@163.com
}

Received January 25, 2012; revised February 27, 2012; accepted March 15, 2012

\begin{abstract}
In this paper, solutions of difference equation $x_{n+1}=\frac{x_{n-1}}{x_{n}}$ for $n=0,1, \cdots$ are investigated, where $x_{-1}, x_{0}$ are both arbitrary nonzero real numbers. The results are applied to the following difference equation $y_{n+1}=\frac{y_{n}-y_{n-1}}{y_{n}-1}$ for $n=0,1, \cdots$.
\end{abstract}

Keywords: Difference Equation; Recursive Sequence; Period-3 Solution

\section{Introduction}

Amleh, Grove and Ladas [1] studied the global stability boundedness character and periodic nature of positive solutions of difference equation

$$
x_{n+1}=\alpha+\frac{x_{n-1}}{x_{n}},
$$

where $\alpha \in[0, \infty)$ and initial conditions $x_{-1}$ and $x_{0}$ are both arbitrary positive real numbers.

Amleh, Grove and Ladas [1] obtain the following theorem.

Theorem A (Amleh, Grove and Ladas [1]) Let $0 \leq \alpha \leq 1$ and $\left\{x_{n}\right\}_{n=-1}^{\infty}$ be a solution of Equation (1) with initial conditions $0<x_{-1} \leq-1$ and $x_{0} \geq \frac{1}{1-\alpha}$.

Then the following statements are true.

1) $\lim _{n \rightarrow \infty} x_{2 n}=\infty$;

2) $\lim _{n \rightarrow \infty} x_{2 n+1}=\alpha$.

Now, we can see that if $x_{-1}=x_{0}=1$ and $\alpha=0$, then $x_{1}=x_{2}=\cdots=1$. So, the theorem A does not hold for $\alpha=0$.

Kulenovic and Glass in their monograph [2] give an open problem as follows.

Open Problem 6.10.7. For the following difference equation determine the "good" set $G \subset \mathbb{R} \times \mathbb{R}$ of the initial conditions $\left(y_{-1}, y_{0}\right) \in \mathbb{R} \times \mathbb{R}$ throng with the equation is well defined for all $n \geq 0$. Then for every $\left(y_{-1}, y_{0}\right) \in G$, investigate the long-term behavior of the solution $\left\{y_{n}\right\}_{n=-1}^{\infty}$ of

$$
y_{n+1}=\frac{y_{n}-y_{n-1}}{y_{n}-1} \text { for } n=0,1, \cdots .
$$

Let $1-y_{n}=x_{n}$. Then Equation (2) can be rewritten as follows

$$
x_{n+1}=\frac{x_{n-1}}{x_{n}},
$$

where $x_{-1}$ and $x_{0}$ are arbitrary nonzero real numbers. To this end, we study Equation (3) and use the results of Equation (3) to Equation (2).

\section{Some Lemmas}

It is easy for one to see that if

$$
A=\left(\begin{array}{ll}
1 & 1 \\
1 & 2
\end{array}\right) \text { and } P=\left(\begin{array}{cc}
1 & 1 \\
\frac{1+\sqrt{5}}{2} & \frac{-1+\sqrt{5}}{2}
\end{array}\right)
$$

then we have

$$
P^{-1} A P=\left(\begin{array}{cc}
\frac{3+\sqrt{5}}{2} & 0 \\
0 & \frac{3-\sqrt{5}}{2}
\end{array}\right)
$$

and

$$
P^{-1}=\left(\begin{array}{cc}
\frac{5-\sqrt{5}}{10} & \frac{\sqrt{5}}{5} \\
\frac{5+\sqrt{5}}{10} & -\frac{\sqrt{5}}{5}
\end{array}\right) .
$$


Lemma 2.1 (Kocic and Ladas [3]) Consider the difference equation

$$
x_{n+1}=F\left(x_{n}, x_{n-1}, \cdots, x_{n-k}\right) \text { for } n=0,1, \cdots .
$$

Assume that $F\left(u_{0}, u_{1}, \cdots, u_{k}\right)$ is a $C^{\prime}$ function and $\bar{x}$ is an equilibrium of Equation (5).

Then the linearized equation associated with Equation (5) about the equilibrium $\bar{x}$ is

$$
y_{n+1}=\sum_{i=0}^{k} \frac{\partial F_{i}}{\partial u_{i}}(\bar{x}, \bar{x}, \cdots, \bar{x}) y_{n-i} \quad \text { for } \quad n=0,1, \cdots
$$

and the following statements are true.

a) If all roots of the polynomial equation

$$
\lambda^{k+1}-\sum_{i=0}^{k} \frac{\partial F_{i}}{\partial u_{i}}(\bar{x}, \bar{x}, \cdots, \bar{x}) \lambda^{k-i}=0
$$

lie in the open unit disk $|\lambda|<1$, then the equilibrium $\bar{x}$ of Equation (5) is asymptotically stable;

b) If at least one root of Equation (6) has absolute value greater than one, then equilibrium $\bar{X}$ of Equation (5) is unstable.

One can refer to Kocic and Ladas [3, Corallary 1.3.2, p14 ].

Lemma 2.2 Equation (3) has two equilibriums $\bar{x}_{1}=0$ and $\bar{x}_{2}=1$.

It is easy to see that $\bar{x}^{2}=\bar{x}$ has two roots and the proof is complete.

\section{Main Results}

Theorem 3.1 Let $x_{-1}=p$ and $x_{0}=q$. Then the following statements are true.

a) $x_{2 n}=\frac{q^{a_{n}}}{p^{b_{n}}}$, where

$$
a_{n}=\frac{5+\sqrt{5}}{10}\left(\frac{3+\sqrt{5}}{2}\right)^{n}+\frac{5-\sqrt{5}}{10}\left(\frac{3-\sqrt{5}}{2}\right)^{n}
$$

and

$$
b_{n}=\frac{\sqrt{5}}{5}\left[\left(\frac{3+\sqrt{5}}{2}\right)^{n}-\left(\frac{3-\sqrt{5}}{2}\right)^{n}\right] \text { for } n=0,1, \cdots .
$$

b) $x_{2 n+1}=\frac{p^{a_{n}}}{q^{b_{n}}}$, where

$$
a_{n}=\frac{5+\sqrt{5}}{10}\left(\frac{3+\sqrt{5}}{2}\right)^{n}+\frac{5-\sqrt{5}}{10}\left(\frac{3-\sqrt{5}}{2}\right)^{n}
$$

and

$b_{n}=\frac{\sqrt{5}}{5}\left[\left(\frac{1+\sqrt{5}}{2}\right)^{2}\left(\frac{3+\sqrt{5}}{2}\right)^{n}-\left(\frac{-1+\sqrt{5}}{2}\right)^{2}\left(\frac{3-\sqrt{5}}{2}\right)^{n}\right]$

for $n=0,1, \cdots$, where $\left\{x_{n}\right\}_{n=0}^{\infty}$ is the solution of Equation (3) with the initial $x_{-1}=p, x_{0}=q$.

Proof: Part a).

Let $x_{-1}=p \neq 0, x_{0}=q \neq 0$. Then by Equation (3) we have

$$
x_{2}=\frac{q^{2}}{p}, x_{4}=\frac{q^{5}}{p^{3}}, x_{6}=\frac{q^{13}}{p^{8}}, \cdots
$$

we assume that

$$
x_{2 n}=\frac{q^{a_{n}}}{p^{b_{n}}} .
$$

Then by induction, we have

$$
\left\{\begin{array}{l}
b_{n+1}=a_{n}+b_{n} \\
a_{n+1}=a_{n}+b_{n+1}=2 a_{n}+b_{n},
\end{array}\right.
$$

where $a_{1}=2, b_{1}=1$ and $n=1,2, \cdots$.

Change Equation (8) into

$$
\left(\begin{array}{c}
b_{n+1} \\
a_{n+1}
\end{array}\right)=\left(\begin{array}{ll}
1 & 1 \\
1 & 2
\end{array}\right)\left(\begin{array}{l}
b_{n} \\
a_{n}
\end{array}\right)
$$

or

$$
Y_{n+1}=A Y_{n},
$$

where $A=\left(\begin{array}{ll}1 & 1 \\ 1 & 2\end{array}\right)$.

From Equation (4), we get

$$
Y_{n+1}=P\left(\begin{array}{cc}
\frac{3+\sqrt{5}}{2} & 0 \\
0 & \frac{3-\sqrt{5}}{2}
\end{array}\right) P^{-1} Y_{n},
$$

where

$$
P=\left(\begin{array}{cc}
1 & 1 \\
\frac{1+\sqrt{5}}{2} & \frac{-1+\sqrt{5}}{2}
\end{array}\right), P^{-1}=\left(\begin{array}{cc}
\frac{5-\sqrt{5}}{10} & \frac{\sqrt{5}}{5} \\
\frac{5+\sqrt{5}}{10} & -\frac{\sqrt{5}}{5}
\end{array}\right) .
$$

Equation (11) can be changed into

$$
P^{-1} Y_{n+1}=\left(\begin{array}{cc}
\frac{3+\sqrt{5}}{2} & 0 \\
0 & \frac{3-\sqrt{5}}{2}
\end{array}\right) P^{-1} Y_{n} .
$$

Let $Z_{n+1}=P^{-1} Y_{n+1}$ and $Z_{n}=P^{-1} Y_{n}$. Then we obtain that

$$
Z_{n+1}=\left(\begin{array}{cc}
\frac{3+\sqrt{5}}{2} & 0 \\
0 & \frac{3-\sqrt{5}}{2}
\end{array}\right) Z_{n}
$$


and

$$
Z_{1}=P^{-1} Y_{n}=P^{-1}\left(\begin{array}{l}
2 \\
1
\end{array}\right)
$$

By induction, we have

$$
Z_{n+1}=\left(\begin{array}{cc}
\left(\frac{3+\sqrt{5}}{2}\right)^{n} & 0 \\
0 & \left(\frac{3-\sqrt{5}}{2}\right)^{n}
\end{array}\right) Z_{1} .
$$

Therefore,

$$
Y_{n+1}=\left(\begin{array}{l}
\frac{\sqrt{5}}{5}\left[\left(\frac{3+\sqrt{5}}{2}\right)^{n}-\left(\frac{3-\sqrt{5}}{2}\right)^{n}\right] \\
\frac{5+\sqrt{5}}{10}\left(\frac{3+\sqrt{5}}{2}\right)^{n}+\frac{5-\sqrt{5}}{10}\left(\frac{3-\sqrt{5}}{2}\right)^{n}
\end{array}\right) .
$$

Hence, the proof of part (a) is complete.

The proof of part (b) can be similarly given, so we omit it. This can complete the proof of theorem 3.1.

By theorem 3.1, we get the following corollary.

Corollary 3.1 Assume that $x_{-1}=p>0, x_{0}=q>0$. Then the following statements are true.

a) If $p=q^{\frac{1+\sqrt{5}}{2}}$, then the positive solution $\left\{x_{n}\right\}_{n=1}^{\infty}$ of Equation (3) converges to 1, i.e, $\lim _{n \rightarrow \infty} x_{n}=1$.

b) If $p>q^{\frac{1+\sqrt{5}}{2}}$, then the positive solution $\left\{x_{n}\right\}_{n=1}^{\infty}$ of Equation (3) has the properties

$$
\lim _{n \rightarrow \infty} x_{2 n}=0 \text { and } \lim _{n \rightarrow \infty} x_{2 n+1}=\infty .
$$

c) If $p<q^{\frac{1+\sqrt{5}}{2}}$, then the positive solution $\left\{x_{n}\right\}_{n=1}^{\infty}$ of Equation (3) has the properties

$$
\lim _{n \rightarrow \infty} x_{2 n}=\infty \text { and } \lim _{n \rightarrow \infty} x_{2 n+1}=0 .
$$

Theorem 3.2 Assume that $x_{-1}=p \neq 0, x_{0}=q \neq 0$. Then the following statements are true.

a) If $p=-1$ and $q=1$, then the solution $\left\{x_{n}\right\}_{n=1}^{\infty}$ of Equation (3) is periodic with period-3 as follows

$$
-1,1,-1,-1,1,-1, \cdots \text {. }
$$

b) If $p=1$ and $q=-1$, then the solution $\left\{x_{n}\right\}_{n=1}^{\infty}$ of Equation (3) is periodic with period-3 as follows

$$
1,-1,-1,1,-1,-1, \cdots \text {. }
$$

c) If $p=-1$ and $q=-1$, then the solution $\left\{x_{n}\right\}_{n=1}^{\infty}$ of Equation (3) is periodic with period-3 as follows

$$
-1,-1,1,-1,-1,1, \cdots \text {. }
$$

The proof of theorem 3.2 is very easy, so we will omit it.

By theorems 3.1 and 3.2, we can obtain the following corollary.

Corollary 3.2 Assume that $\left(x_{-1}, x_{0}\right) \in \mathbb{R}^{2} /(0,0)$. Then the following statements are true.

a) If $|p|=|q|^{\frac{1+\sqrt{5}}{2}}$ and at least one of $p$ and $q$ is less than 0 , then $\left\{x_{n}\right\}_{n=1}^{\infty}$ of Equation (3) converges to a period-3 solution of Equation (3) as one of (10)-(12).

b) If $|p|>|q|^{\frac{1+\sqrt{5}}{2}}$ and at least one of $p$ and $q$ is less than 0 , then $\left\{x_{n}\right\}_{n=1}^{\infty}$ of Equation (3) has the following properties

$$
\lim _{n \rightarrow \infty} x_{2 n}=0 \text { and } \lim _{n \rightarrow \infty}\left|x_{2 n+1}\right|=\infty .
$$

c) If $|p|<|q|^{\frac{1+\sqrt{5}}{2}}$ and at least one of $p$ and $q$ is less than 0, then $\left\{x_{n}\right\}_{n=1}^{\infty}$ of Equation (3) has the following properties

$$
\lim _{n \rightarrow \infty}\left|x_{2 n}\right|=\infty \text { and } \quad \lim _{n \rightarrow \infty} x_{2 n+1}=0 .
$$

d) If at least one of $p$ and $q$ is less than 0 , then every solution of Equation (3) strictly oscillates about the equilibrium $\bar{x}=0$.

e) If $|p| \neq|q|^{\frac{1+\sqrt{5}}{2}}$ and at least one of $p$ and $q$ is less than 0 , then every solution of Equation (3) strictly oscillates about the equilibrium $\bar{x}=1$.

Theorem 3.3 The equilibrium $\bar{x}=1$ of Equation (3) is unstable.

Proof: The linearize equation associated with Equation (3) about the equilibrium $\bar{x}=1$ is

$$
\mathrm{y}_{n+1}=-y_{n}+y_{n-1} .
$$

The characteristic Equation of (17) is

$$
\lambda^{2}+\lambda-1=0
$$

Thus, we obtain two roots $\lambda_{1,2}=\frac{-1 \pm \sqrt{5}}{2}$. Noting that $\left|\lambda_{2}\right|=\left|\frac{-1-\sqrt{5}}{2}\right|>1$. Therefore, by lemma 3.1, we know that the equilibrium $\bar{x}=1$ of Equation (3) is unstable. The proof of theorem 3.3 is complete.

\section{Application}

By theorem 3.1, we have the following theorem.

Theorem 4.1 Assume that $y_{-1}<1$ and $y_{0}<1$. Then the following statements are true.

a) Every solution $\left\{y_{n}\right\}_{n=1}^{\infty}$ of Equation (2) satisfies 
$\mathrm{y}_{n}<1$ for $n=-1,0,1, \cdots$

b) If $1-y_{-1}=\left(1-y_{0}\right)^{\frac{1+\sqrt{5}}{2}}$, then the solution $\left\{y_{n}\right\}_{n=1}^{\infty}$ of Equation (2) converges to 0 .

c) If $1-y_{-1}>\left(1-y_{0}\right)^{\frac{1+\sqrt{5}}{2}}$, then the solution $\left\{y_{n}\right\}_{n=1}^{\infty}$ of Equation (2) has the following properties

$$
\lim _{n \rightarrow \infty} y_{2 n}=1 \text { and } \lim _{n \rightarrow \infty} y_{2 n+1}=-\infty \text {. }
$$

d) If $1-y_{-1}<\left(1-y_{0}\right)^{\frac{1+\sqrt{5}}{2}}$, then the solution $\left\{y_{n}\right\}_{n=1}^{\infty}$ of Equation (2) has the following properties

$$
\lim _{n \rightarrow \infty} y_{2 n}=-\infty \text { and } \lim _{n \rightarrow \infty} y_{2 n+1}=1 \text {. }
$$

By corollary 3.2, we get the following theorem.

Theorem 4.2 Assume that $\left(y_{-1}, y_{0}\right) \in \mathbb{R}^{2} /(1,1)$. Then the following statements are true.

a) If $\left|y_{-1}-1\right|=\left|y_{0}-1\right|^{\frac{1+\sqrt{5}}{2}}$ and at least one of $1-y_{-1}$ and $1-y_{0}$ is less than 0 , then $\left\{y_{n}\right\}_{n=1}^{\infty}$ of Equation (2) converges to a period-3 solution of Equation (2) as one of the following:

i) $2,0,2,2,0,2, \cdots$,

ii) $0,2,2,0,2,2, \cdots$,

iii) $2,2,0,2,2,0, \cdots$,

b) If $\left|y_{-1}-1\right|>\left|y_{0}-1\right|^{\frac{1+\sqrt{5}}{2}}$ and at least one of $1-y_{-1}$ and $1-y_{0}$ is less than 0 , then every solution $\left\{y_{n}\right\}_{n=1}^{\infty}$ of Equation (2) has the following properties:

$$
\lim _{n \rightarrow \infty} y_{2 n}=1 \text { and } \lim _{n \rightarrow \infty}\left|y_{2 n+1}\right|=\infty \text {. }
$$

c) If $\left|y_{-1}-1\right|<\left|y_{0}-1\right|^{\frac{1+\sqrt{5}}{2}}$ and at least one of $1-y_{-1}$ and $1-y_{0}$ is less than 0 , then every solution $\left\{y_{n}\right\}_{n=1}^{\infty}$ of Equation (2) has the following properties:

$$
\lim _{n \rightarrow \infty}\left|y_{2 n}\right|=\infty \text { and } \quad \lim _{n \rightarrow \infty} y_{2 n+1}=1 \text {. }
$$

d) If $\left|y_{-1}-1\right| \neq\left|y_{0}-1\right|^{\frac{1+\sqrt{5}}{2}}$ and at least one of $1-y_{-1}$ and $1-y_{0}$ is less than 0 , then every solution $\left\{y_{n}\right\}_{n=1}^{\infty}$ of Equation (2) strictly oscillates about the equilibrium $\bar{y}=0$ of Equation (2).

\section{Acknowledgements}

Research supported by Distinguished Expert Foundation and Youth Science Foundation of Naval Aeronautical and Astronautical University.

\section{REFERENCES}

[1] A. M. Amleh, E. A. Grove and G. Ladas, "On the Recursive Sequence $x_{n+1}=\alpha+\frac{x_{n-1}}{x_{n}}$," Journal of Mathematical Analysis and Applications, Vol. 233, No. 2, 1999, pp. 790-798. doi:10.1006/jmaa.1999.6346

[2] M. R. S Kulenovic and L. Glass, "Dynamics of Second Order Rational Difference Equations,” Chapman Hall/ CRC, USA, 2002.

[3] V. L. Kocic and G. Ladas, "Global Asymptotic Behavior of Nonlinear Difference Equation of Higher Order with Applications," Kluwer Academic, Dordrecht, 1993. 